\title{
Role of the Mode Theory on Lightning Generated Waves Propagating in Venus-Ionosphere Waveguide
}

\author{
R. N. SINGH ${ }^{1}$ and Hari Om UPADHYAY ${ }^{2}$ \\ ${ }^{1}$ Department of Applied Physics, Institute of Technology, Banaras Hindu University, Varanasi - 221 005, India \\ ${ }^{2}$ Physics Department, Faculty of Science, Banaras Hindu University, Varanasi - 221005 , India
}

(Received June 18, 1990; Revised February 18, 1992)

\begin{abstract}
The wave mode either electromagnetic or electrostatic of the OEFD measured signals is unknown and needs to be ascertained by indirect means. Considering these waves to be generated by global Venus lightning, the wave attenuation along the Venus-ionosphere wave-guide has been computed. The effective wave attenuation versus frequency shows a "knee" characterizing minimum attenuation $6 \mathrm{kHz}$. This "knee" is consistent with the amplitude spectral peak of OEFD signals around this frequency. This feature of the OEFD signals implies that the Venus lightning may have global occurrence. The lightning generated signals may travel considerable distance within the Venus-ionosphere waveguide. The encounter of these signals with plasma "holes" or ion "troughs" in the nightside of Venus wake region results in the leakage of the signals out of the shielding ionosphere. These signals are occasionally recorded by the OEFD. The occurrence rate statistics shows that the leakage of $5.4 \mathrm{kHz}$ signals through the plasma "holes" in the vicinity of PVO periapsis are most effective. The maximum occurrence rate of higher frequency signals change in altitude slightly from one PVO season to another.
\end{abstract}

\section{Introduction}

The generation of electromagnetic waves covers a broad band spectrum which decreases faster in strength at lower frequencies and decreases rather slowly and extending upto $100 \mathrm{MHz}$ (KIMPARA, 1965). The lightning process in Venus atmosphere is under debate. The first evidence of lightning came from in situ measurements aboard Venera-11 (KSANFOMALITY, 1979, 1980). These measurements were also made at four different frequencies namely 10, 18, 38 and $80 \mathrm{kHz}$. The Pioneer Venus Orbiter (PVO) was launched with rather simple electric field detector (OEFD) operating at four spot frequencies; $100 \mathrm{~Hz}, 730$ $\mathrm{Hz}, 5.4 \mathrm{kHz}$ and $30 \mathrm{kHz}$ (SCARF et al., 1980). The OEFD recorded signals are contaminated by the Sun's light and is not able to give reliable measurements of electric field during the dayside sector of the Venus atmosphere. Since OEFD instrument basically measures the electric field strength at four different frequencies, the exact nature of these signals whether electrostatic or electromagnetic is to be inferred by some additional indirect features of these signals. The wave modes of such signals are closely related with the generation mechanism and their propagation properties.

The OEFD measures strong impulsive wave bursts whenever PVO nightside priapsis is low. Various features of $100 \mathrm{~Hz}$ signals led TAYLOR et al. (1979) and SCARF et al. (1980) to find a close resemblance with the earlier in situ measurement of electromagnetic noise using Venera-11 lander which were attributed to Venus lightning (KSANFOMALITY, 1979, 1980). Since PVO does not descend below about $140 \mathrm{~km}, 100 \mathrm{~Hz}$ signal was considered to propagate upwards along the nightside wake region magnetic field in whistler mode. The occurrence statistics revealed some clustering and it was found to correlate well with the highland topography of Venus (SCARF and RUSSELL, 1983). This correlation further implied that the enhanced occurrence rates of $100 \mathrm{~Hz}$ signal may be stimulated by active volcanoes in the Venus highlands. This interpretation of SCARF and RUSSELL (1983) was questioned by TAYLOR et al. (1985) and alternative suggestion was made that these waves are correlated with ion density "troughs" which are of localised in the midnight meridian. The generation of these localized waves was attributed by TAYLOR $e t$ 
al. (1985) to "the complicating interaction between the solar wind and the nightside Venus ionosphere". SINGH and RUSSELL (1986) analysed the OEFD data rather closely and noted that despite $100 \mathrm{~Hz}$ signals, the higher frequency signals $730 \mathrm{~Hz}, 5.4$ and $30 \mathrm{kHz}$, at times, exhibit simultaneity and more or less resemblance with the impulsive nature of $100 \mathrm{~Hz}$ signals. These signals were interpreted to be propagating upwards whenever the ideal conditions of geometrical optics resulting into specular reflections were violated in the wake region of the Venus ionosphere.

TAYLOR and CLOUTIER (1987) and TAYLOR et al. (1987) continued questioning the lightning interpretation of OEFD measured signals and have viewed this interpretation in terms of deeper question whether Venus is "dead or alive". However, no serious effort has been made by these authors to unfold the earlier suggestion. The detailed statistical study of occurrence rates of OEFD signals have shown beyond doubt that the impulsive wave bursts appear on all the four frequencies and these impulsive signals are different from the monotonic and conspicuous interference signals which appear at times (RUSSELL et al., 1980a, b, c). The statistical analysis of OEFD signal stands out beyond any room for further confusion with telemetry interference signals. The existing provision of two antennas on OEFD were used to show that these impulsive signals are partially polarized which further supports that these waves are electromagnetic waves (SCARF and RUSSELL, 1988).

If these signals are generated by the Venus lightning, it must conform to the global picture of lightning as is well known in the case of lightning in the earth's atmosphere. The lightning in the Venus cloud must take place during the dayside as well as nightside. In this paper, we have interpreted these signals to be generated by global Venus lightning. It is shown that the lightning generated signals propagate through the Venus-ionosphere waveguide. Using waveguide propagation and taking into account the nature of boundaries and the filling gas, we have further shown that the $6 \mathrm{kHz}$ signals suffer minimum attenuation through the Venus-ionosphere waveguide. The waveguide is highly asymmetric with minimum height in the dayside region and wake region of the Venus ionosphere is highly inhomogeneous with the nightside varying height of the upper boundary with holes and troughs which may allow the occasional upwelling of the lightning signals propagating through the waveguide. The occurrence rate analysis of the OEFD signals is therefore mixed up and, at time, it may have significant fraction of dayside lightning signals which propagate to the nightside and are partially transmitted through the plasma "holes". As such these "holes" and "trough" may behave somewhat like slotted waveguide which is now extensively used as radiators at microwave frequencies.

\section{Solar Wind Interaction with Venus and Formation of Wake Region}

The morphological changes in the dayside and nightside ionosphere of Venus is one of the important features in the propagation of lightning signals. The dayside ionospheric boundary is somewhat denser and lower as compared to nightside. The detailed height profiles of some of these parameters are illustrated in Section 3. These features are schematically shown in Fig. 1. Important features relevant to lightning study is the wake region of Venus with hung-up magnetic field and formation of plasma density inhomogeneities discussed in details by BRACE et al. $(1982,1983)$ and by TAYLOR et al. (1979). These nightside features helps the escape of lightning signals easier as compared dayside ionosphere. The escaping probability of higher frequency signals increases and it is quite likely that the lightning signals could be mapped all around the planet. The planned flyby of Galileo past Venus and detection or nodetection would provide an important clue about Venus lightning. Correlation of OEFD signals with nightside wake region has been reported extensively. However, we shall use some of the illustrated features for waveguide mode propagation of lightning generated signals and their partial transmission through the plasma density "holes" and "troughs" shown in Fig. 1.

\section{Detailed Features of Waveguide Boundaries and Wave Generation}

The Venera and PVO missions have provided adequate information about the Venus ionospheric 


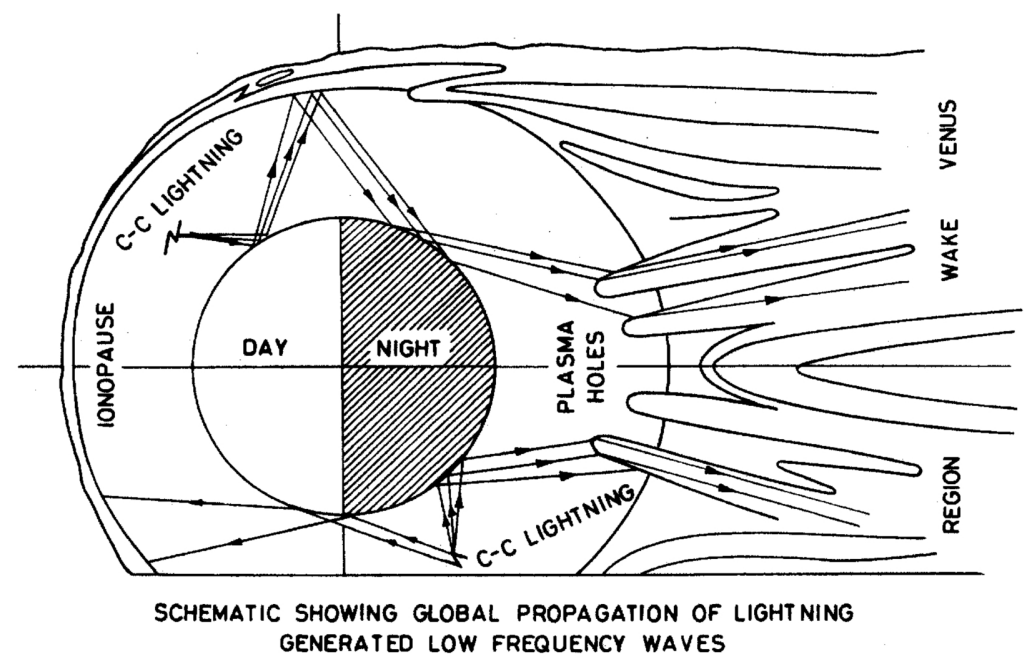

Fig. 1. Schematic showing the waveguide mode propagation of Venus lightning generated signals and their leakage upwards through the plasma "holes" having radial magnetic field configuration.

parameters that are generally needed for the study of wave propagation. In this section, we present the electron density and magnetic field profiles of nightside (orbit Nos. 22, 515, 535, 562, $576 \mathrm{IN}$, and OUT, 577 IN and OUT, and 578) PVO orbits in Figs. 2(a) and (b) and dayside (orbit Nos. 176, 207, 209, 211, 383, 385, 408, 412 and 417) PVO orbits in Figs. 3(a) and (b). The hatched regions of these profiles have been used in computing the height profiles of electron collision frequencies. Using formulations of collision frequencies (SCHUNK and NAGY, 1980), the collision frequency height profiles for dayside and nightside Venus ionosphere have been computed and shown in Fig. 4. The computed height profiles clearly show that the upper boundary of the Venus-ionosphere waveguide is only partially conducting. In addition, the nightside ionospheric electron density is comparatively lower than the dayside electron density. Because of the turbulent wake region, the nightside ionosphere is quite irregular and consists of hungup magnetic field regions forming electron density "holes" and ion "troughs" as shown in Fig. 1 (BRACE et al., 1982). These regions are known to transmit the lightning signals upward which is detected whenever the spacecraft passes over these regions during these regions. Under suitable conditions, the lightning signals at all the four frequencies have been recorded by OEFD aboard PVO. The surface properties of Venus are not adequately known. The surface temperature is larger than the earth's surface, the moisture content is also small and the surface is distributed with high mountains, craters and volcano sites. It is not precisely known whether the volcanoes are currently active. For purposes of wave propagation by multiple reflections within the Venus-ionosphere waveguide, in this paper, we assume the Venus surface conductivity to be infinite. The differential conductivity of upper boundary depends on the varying parameters of the ionospheric region.

The process of lightning in Venus clouds is a much more complex problem. The cloud deck is dense, hot and dynamical features seem to be quiet. It is therefore not known how the charge separation in the Venus clouds takes place. Since the height of the Venus' cloud deck is at a height of about $55 \mathrm{~km}$ and is $15-20 \mathrm{~km}$ thick, it is almost certain that the cloud-to-cloud discharge are the source of lightning signals. The cloud composition and prevailing dynamics would be helpful in the understanding of lightning generation in the Venus clouds. Another possibility of lightning generation lies in the proposed distribution of active volcanoes on the high mountains (SCARF and RUSSELL, 1983). With the present state of data availability, it is not all possible to pin down the problem of lightning generation. However, the Venera lander (KSANFOMALITY et al., 1983) and PVO data (TAYLOR et al., 1979; SiNGH and RUSSELL, 

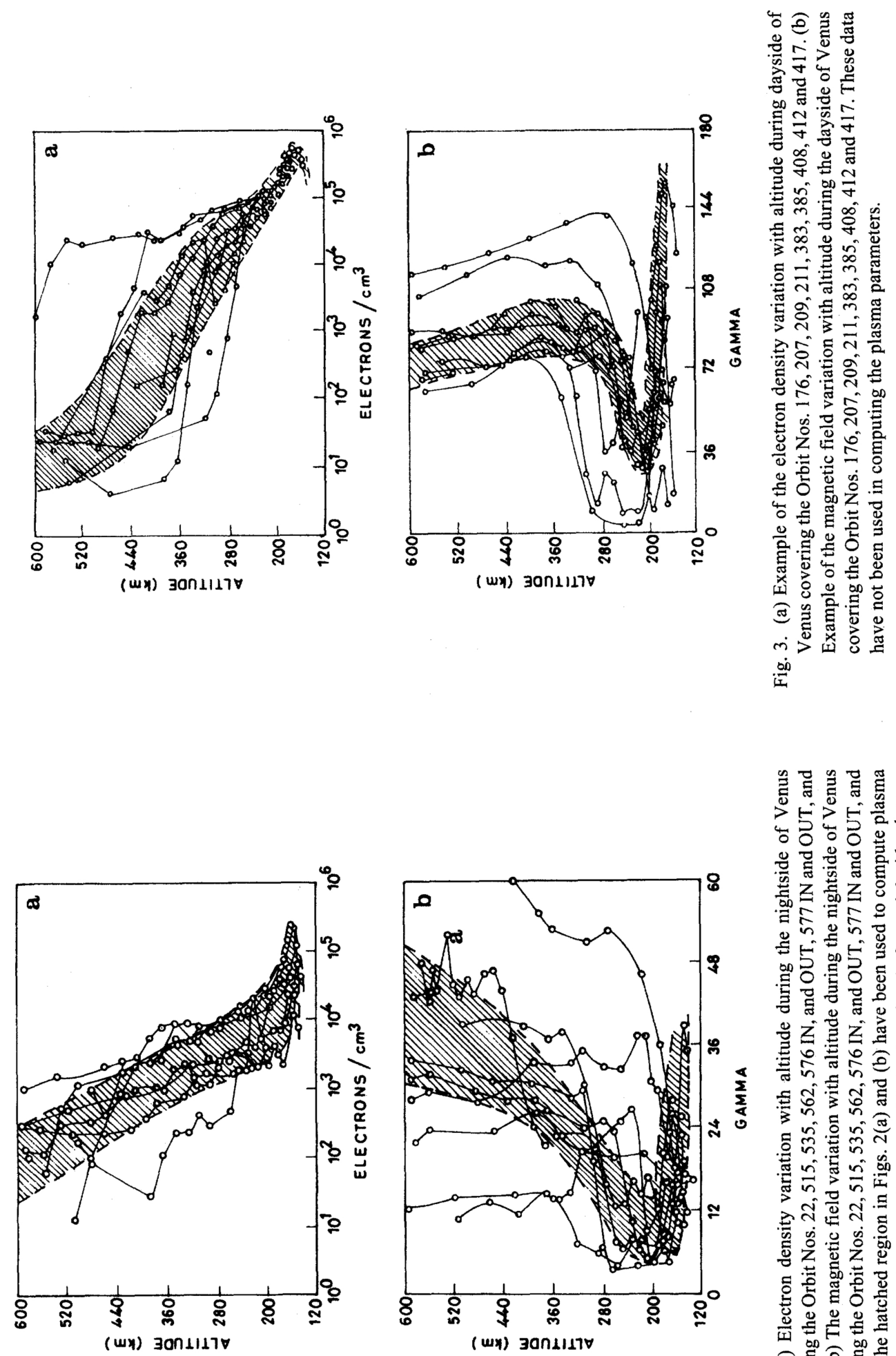

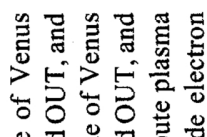

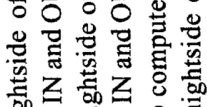

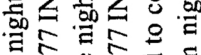

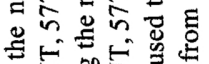

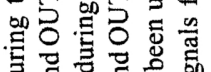

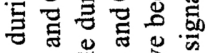

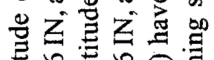

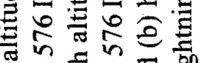

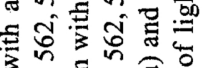

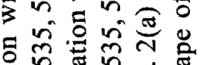

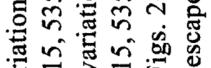

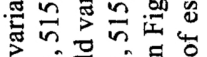
ิำษ ำ 중

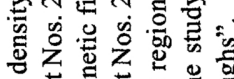

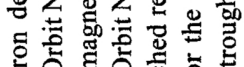
冚

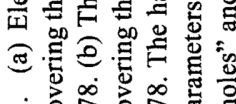

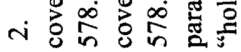
䁙 


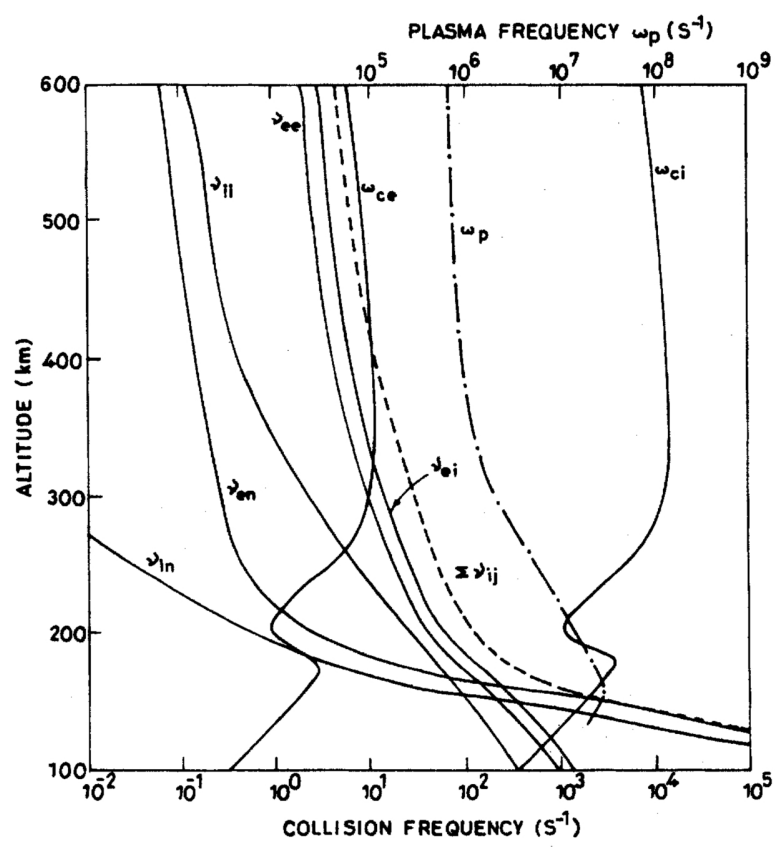

Fig. 4. Variation of various collision frequencies, electron gyrofrequency, ion gyrofrequency and electron plasma frequency with altitude using the data shown in Figs. 2(a) and (b).

1986; RUSSELL et al., 1988a, b, c) have putforward pressing information to believe that the Venus lightning does take place.

\section{Propagation and Attenuation of Lightning Generated Waves}

The electromagnetic wave propagation between the two spherical parallel plates is generally described in terms of mode theory (BREMMER, 1949; BUDDEN, 1952; WAIT, 1957). The VLF waves reflecting between the parallel spherical shell formed by the Venus lower ionospheric layer and the Venus surface propagate in various modes satisfying the transcedental wave mode equation (WAIT, 1957).

$$
R_{\mathrm{i}} R_{\mathrm{v}}=\exp \left(+i 4 \pi h C_{n} / \lambda\right) \cdot \exp (-i 2 \pi n)
$$

where $h$ is the height between Venus spherical surface and the Venus spherical reflecting ionospheric layer, $\lambda$ is the wavelength of the propagating waves, the cosine of the complex angle of incidence is denoted by $C_{n}$ and $n=0,1,2, \ldots, n$ define various modes of propagation of lightning generated electromagnetic waves. The lefthand side of Eq. (1) is the product of reflection coefficients $R_{\mathrm{v}}$ and $R_{\mathrm{i}}$ which refer to the Venus surface and the Venus ionospheric layer respectively. The approximate solution of the wave mode Eq. (1) for the parallel guiding spherical shell formed by the Earth's surface and the Earth's ionospheric has been obtained by WAIT (1957). With relevant change of parameters, the solution of Eq. (1) can also be used in the case of guiding shell formed by the Venus surface and its ionosphere. The Venus surface is considered to be perfectly reflecting $(\sigma \rightarrow \infty)$ and the ionospheric reflection to be partial which is governed by the varying collision frequency $v$ and the plasma frequency $\omega_{\mathrm{p}}$ of the Venus lower ionospheric layer. In the case of Venus, the cloud-to-cloud discharges radiate electromagnetic waves with a dipolar power distribution. Taking asymptotatic expansion for the Legendre function, the maximum electromagnetic wave radiation from a horizontal radiating electric dipole at a radial distance " $d$ " within 
the parallel spherical guiding shell is written as the mode sum (WAIT, 1957)

$$
E=E_{0} W \mathrm{mVm}^{-1}
$$

where $E_{0}$ is the electric field radiated by a lightning stroke considered as a vertical dipole here. If the radiated power $P$ is expressed in kilowatts and the radial distance from an assumed vertical lightning discharge $d$ (expressed in kilometer), the electric field is written as

$$
E_{0}=\frac{300 \sqrt{P}}{d} \mathrm{mVm}^{-1}
$$

The dimensionless factor $W$ in Eq. (2) governs the changes taking place in the signals while propagating through the waveguide. The signal attenuation is also contained in the dimensionless factor and also depends on various waveguide parameters and the complex functions of angles satisfying the wave mode Eq. (1).

$$
W=\left[\frac{d / a}{\sin d / a}\right]^{1 / 2} \frac{(d / \lambda)^{1 / 2}}{h / \lambda}\left|\sum_{n=0}^{\infty} \delta_{n} S_{n}^{3 / 2} e^{-i 2 \pi S} n^{(d / \lambda)}\right|
$$

where $h$ and $\lambda$ are expressed in kilometers $d \simeq a \theta, a$ is the Venus radius in $\mathrm{km}$

$$
\begin{aligned}
\delta_{n}=\left[1+\sin \left(2 k h C_{n}\right) / 2 k h C_{n}\right]^{-1} & \simeq 1 / 2 & & \text { for } n=0 \\
& \simeq 1 & & \text { for } n=1,2,3, \ldots .
\end{aligned}
$$

The waveguide mode attenuation of the lightning signal is mainly governed by the exponential term in Eq. (4). The complex sine function appearing in the exponential when separated in real and imaginary parts are written as

$$
\begin{aligned}
& \operatorname{Re}_{n}=\bar{S}_{n}+\frac{1}{2^{3 / 2} \pi(h / \lambda) \bar{S}_{n}}\left[\frac{(n-1 / 2)^{2}}{(2 h / \lambda)^{2}}\left\{L^{1 / 2}-\frac{1}{L^{1 / 2}}\right\}\right] \\
& \operatorname{Im} S_{n}=-\frac{1}{2^{3 / 2} \pi(h / \lambda) \bar{S}_{n}}\left[\frac{(n-1 / 2)^{2}}{(2 h / \lambda)^{2}}\left\{L^{1 / 2}+\frac{1}{L^{1 / 2}}\right\}\right] \\
& \bar{S}_{n}=\left(1-C_{n}^{2}\right)^{1 / 2}=\left[1-(n-1 / 2)^{2} /\left(\frac{2 h}{\lambda}\right)^{2}\right]^{1 / 2}
\end{aligned}
$$

and

$$
L=\frac{\omega v}{\omega_{\mathrm{p}}^{2}}
$$




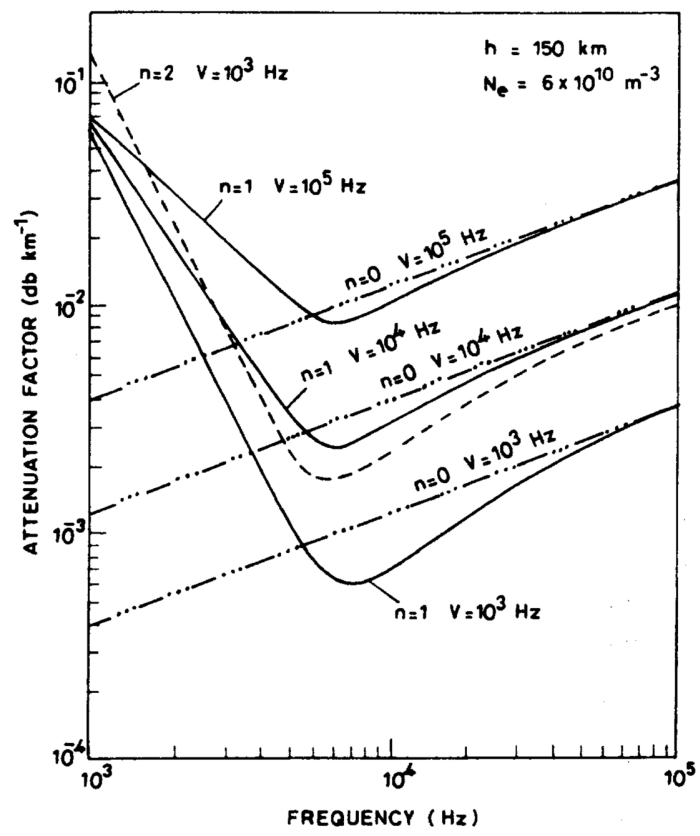

Fig. 5. Variation of attenuation factor computed using mode theory for VLF wave propagation through the Venus-ionosphere waveguide. The wave attenuation shows a minimum attenuation for, $n>0$ modes around $f \simeq 6 \mathrm{kHz}$.

The attenuation of lightning generated signals satisfying the wave mode equation in decibels per kilometer of propagation path in the Venus-ionosphere waveguide is thus written as

$$
\alpha_{n}=-8.68 \times \frac{2 \pi}{\lambda} \operatorname{Im}\left(S_{n}\right) \quad n=0,1,2, \ldots, n .
$$

The variation of wave attenuation with frequencies satisfying the wave mode Eq. (1) for infinite Venus surface conductivity and different values of ionospheric parameters as described and discussed in Section 3 and for different modes of waveguide propagation has been computed and results are shown in Fig. 5 .

\section{Results and Discussion}

It is well known that the lightning produces a wideband frequency spectrum with a field intensity peak lying in VLF range. The radiated intensity of the lightning signals decrease faster at lower frequencies $\left(f^{1}\right)$ as compared to $f^{2}$ variation at higher frequencies. If Venus lightning takes place, in all probability, it would be a global phenomena. This implies that the VLF signals generated by the lightning process would also be propagating globally through the Venus-ionosphere waveguide. Thus it is quite obvious that the lightning signals generated in the dayside region of Venus cloud top can propagate through the waveguide to nightside sector of Venus and vice-versa. The nightside ionosphere consists of plasma "holes" and ion "troughs" which manifest the escape of the signals upwards (SINGH et al., 1987). In view of the schematic shown in Fig. 1 some important facts emerge out: (i) We find that invariably the signals generated from the lightning source situated any where propagate through the waveguide before these signals encounter plasma "holes" in the nightside region of the Venus ionosphere. (ii) Each of these events are characterized by varying degree of wave mode attenuation through the waveguide. (iii) $\mathrm{The} 100 \mathrm{~Hz}$ signals are below the waveguide cut off do not satisfy the waveguide mode propagation and are localized. 
In addition the $100 \mathrm{~Hz}$ waves are capable of escaping out of the Venus ionosphere in the whistler mode. (iv) Since lightning sources are necessarily not just below the observation sites, only a limited number of carefully chosen $100 \mathrm{~Hz}$ events can be used for extrapolating their propagation path and seeking their correlation with the highland topography of Venus. (v) It appears that the impulsive wave bursts events obtained from OEFD wave data are only a small fraction of the total global Venus lightning events. The occurrence rate statistics should therefore be carefully used for comparing it with the observed terrestrial lightning rate. The $100 \mathrm{~Hz}$ signals have been studied extensively (SCARF and RUSSELL, 1983; RUSSELL et al., 1988b; TAYLOR et al., 1979). The occurrence rate of these signals is comparatively larger than the occurrence rates of signals at other three frequencies namely $730 \mathrm{~Hz}, 5.4$ and $30 \mathrm{kHz}$. It has been argued that the magnitude and direction of the magnetic field in the nightside of Venus ionosphere is such that the $100 \mathrm{~Hz}$ signals could escape out of the ionosphere in the whistler mode. In addition to $100 \mathrm{~Hz}$ signals, simultaneous occurrence of impulsive bursts on all the four OEFD frequencies have been observed (SINGH and RUSSELL 1986; RUSSELL et al., 1988a). The geometrical optics approach does not allow the propagation of electromagnetic waves of frequency $\omega$ out of the ionospheric region whose plasma frequency $\omega_{\mathrm{p}}>\omega$. This was one of the reasons for ignoring higher frequency signals in the interpretation of OEFD signals generated from Venus' cloud-to-cloud discharges. It is well known that the nightside of Venus ionosphere contains plasma density inhomogeneities in the form of electron density "holes" or ion density "troughs". In such a case specular reflection of the signals may not take place and the incident signals are partially transmitted upwards. The upward transmission of incident signals depends chiefly on the scale length distribution of inhomogeneities. An outline of mechanism of partial transmission of waves illuminating the plasma inhomogeneity or plasma "hole" has been discussed by SINGH et al. (1987).

The attenuation of the lightning signals in the frequency range $1-30 \mathrm{kHz}$ for two values of the $L$ parameters and for $n=0,1,2$ have been computed and its variations are shown in Fig. 5. The attenuation for $n=0$ wave mode is minimum at $1 \mathrm{kHz}$ and is seen to increase with increasing frequencies. The change of collision frequency is shown to affect the wave attenuation significantly. For $n=1,2$, the wave at-

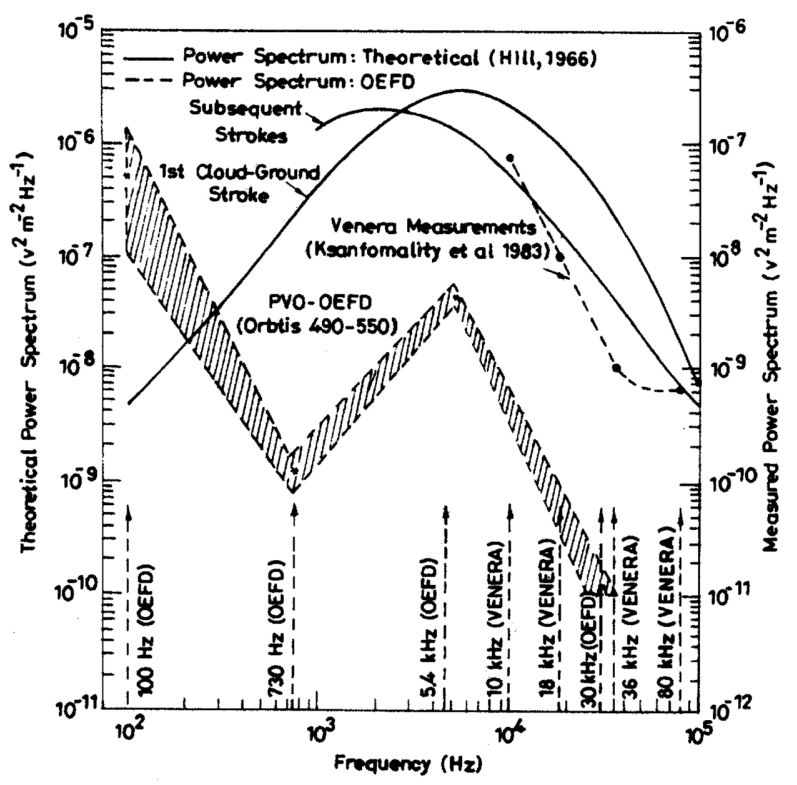

Fig. 6. Power spectral distribution of OEFD signals showing a peak around $f \simeq 6 \mathrm{kHz}$. The signal at low frequencies show further increase because of its unattenuated escape out of the Venus ionosphere in whistler mode. Comparison with theoretical and experimental results shown by SINGH and RUSSELL (1986). 
tenuation shows a "knee" with a minimum attenuation. The wave attenuation around this "knee" is seen to increase with increasing as well as decreasing frequencies. The maximum in the wave amplitude spectrum of OEFD signals was also found to appear around this frequency. The measured and theoretical signal strength was found to be maximum corresponding to this low attenuation which is seen to decrease on either side of this peak as reproduced from (SINGH and RUSSELL, 1986) and shown in Fig. 6. Exception to general attenuation at $100 \mathrm{~Hz}$ is well known. This feature of the $100 \mathrm{~Hz}$ OEFD signal arises because of its unattenuated escape out of the Venus ionosphere in whistler mode (SINGH and RUSSELL, 1986). The appearance of amplitude spectral peak around same frequency from theoretical wave attenuation study as well as from the analysis of OEFD signals demonstrates the validity of waveguide mode propagation of lightning generated signals. Since the dayside lightning events were not recorded by OEFD with contamination of dayside signal with Sun's light, most of the results reported so far have projected the OEFD detected signals as nightside phenomena. It is unfortunate that the dayside OEFD data which is capable of either proving or disproving some of the important points regarding the basic wave mode (electromagnetic or electrostatic) is not available.

The analyses of OEFD wave data exhibit many more features of lightning generated signals that cannot be attributed to signals generated by another source. The main limitation of the OEFD data is that some of the wave features observed, inferred and believed cannot be elegantly proved to ascertain the Venus lightning as a source. The high frequency airborne mapping using RAE1 satellite has been used to study the global features of terrestrial lightning (STONE, 1973). Highly sophisticated high frequency wave detector has been flown aboard Galileo which is scheduled to flyby Venus. This instrument although would flyby Venus and is capable of detecting the high frequency signals only for a short duration proving or disapproving the existence of lightning on Venus. However, an important feature whether Venus lightning is of global character or not can only be established by another Venus Orbiter program.

\section{REFERENCES}

Brace, L. H., R. F. Theis, H. G. Mayr, S. A. Curtis, and J. G. Luhmann, Holes in the nightside ionosphere of Venus, J. Geophys. Res., 87, 199-211, 1982.

Brace, L. H., H. A. TAYLOR, Jr., T. I. Gombosi, A. J. Kliore, W. C. KNUdSEN, and A. F. NAGY, The ionosphere of Venus: Observation and their interpretation, in Venus, edited by D. M. Hunten et al., pp. 779-840, The University of Arizona Press, 1983.

Bremmer, H., Terrestrial Radio Waves, Elsevier Publishing Company, Amsterdam, Netherlands, 1949.

Budden, K. G., The propagation of radio atmospherics, Phil. Mag., 43, 1179-1200, 1952.

HILL, R. D., Electromagnetic radiation from return stroke in a lightning dicharge, J. Geophys. Res., 71, $1963-1967,1966$.

KIMPARA, A., Electromagnetic energy radiated from lightning, in Problems of Atmos. and Space Electricity, edited by S. C. Coronity, p. 532, Elsevier, Amsterdam, 1965.

Ksanfomality, L. V., Lightning in the Venus clouds layer, Cosmic Res., 17, 747-762, 1979.

KSANFOMALITY, L. V., Discovery of frequent lightning discharges in clouds on Venus, Nature, 284, 244-246, 1980.

KSANFomality, L. V., F. L. SCARF, and W. W. L. TAYLOR, The electrical activity of the atmosphere of Venus, in Venus, edited by D. M. Hunten et al., pp. 565-603, University of Arizona Press, Tucson, 1983.

Russell, C. T., M. VON DORNUM, and F. L. SCARF, The altitude distribution of impulsive signals in the night ionosphere of Venus, J. Geophys. Res., 93, 5915-5921, 1988a.

Russell, C. T., M. VON DORnUM, and F. L. SCARF, Planetographic clustering of low altitude impulsive electric signals in the night ionosphere of Venus, Nature, 331, 591-594, $1988 \mathrm{~b}$.

Russell, C. T., M. VON DoRnum, and F. L. SCARF, VLF bursts in night ionosphere of Venus: Effect of magnetic field, Planet. Space Sci., 36, 1211-1218, 1988c.

SCARF, F. L. and C. T. Russell, Lightning measurements from the Pioneer Venus Orbiter, Geophys. Res. Lett., 10, 1192-1195, 1983.

SCARF, F. L. and C. T. Russell, Evidence for lightning and volcanic activity on Venus, Science, 240, 222-224, 1988.

SCARF, F. L., W. W. L. TAYLOR, and L. H. BRACE, Lightning on Venus: Orbiter detection of whistler signals, J. Geophys. Res., 85, 5915-5921, 1980.

SCHUNK, R. W. and A. F. NAGY, Ionospheres of the terrestrial planets, Rev. Geophys. Space Phys., 18, 813-852, 1980.

SINGH, R. N. and C. T. Russell, Further evidence for lightning on Venus, Geophys. Res. Lett., 13, 1051-1054, 1986.

Singh, R. N., C. T. Russell, and F. L. SCARF, Partially transmitted lightning signals recorded by Pioneer Venus Orbiter, $A d v$. Space Res., 7(12), 285-289, 1987. 
Stone, R. G., Radio physics of the outer solar system, Space Sci. Rev., 14, 534-551, 1973.

TAYLOR, H. A., Jr. and P. A. Cloutier, Comment on further evidence for lightning at Venus, Geophys. Res. Lett., 14, 568-570, 1987.

TAYlor, H. A., Jr., J. M. Grebowsky, and P. A. Cloutier, Venus nightside ionospheric troughs: Implication for evidence of lightning and volcanism, J. Geophys. Res., 90, 7415-7426, 1985.

TAYLOR, H. A., Jr., P. A. Cloutier, and Z. ZHENG, Venus lightning signals reinterpreted as in situ plasma noise, J. Geophys. Res., 92, 9907-9919, 1987.

TAYlOR, W. W. L, F. L. SCARF, C. T. RusSELL, and L. H. BRACE, Evidence for lightning on Venus, Nature, 279, 614-616, 1979.

WAIT, J. R., The mode theory of VLF ionospheric propagation for finite ground conductivity, Proc. I.R.E., 45, 760-767, 1957. 